\title{
Cam-type FAI: is the alpha angle the best MR arthrography has to offer? (Skeletal Radiol. 2009;38(9):855-62)
}

\author{
Derek G. Lohan • Leanne L. Seeger • \\ Kambiz Motamedi • Sharon Hame • James Sayre
}

Received: 3 November 2009 / Accepted: 3 November 2009/Published online: 26 November 2009

(C) The Author(s) 2009. This article is published with open access at Springerlink.com

The authors welcome this opportunity to respond to and clarify a number of issues raised by the letter of Drs. Beaulé and Rakhra in relation to our recent paper entitled "Camtype FAI: is the alpha angle the best MR arthrography has to offer?" [1]

Drs. Beaulé and Rakhra express concern regarding our use of intra-operative femoral head-neck osteochondroplasty/arthroscopic debridement in differentiating between the presence or absence of cam-type femoral-acetabular impingement (FAI). Specifically, they refer to the potential of the introduction of significant observer bias using this approach, drawing particular attention to the discrepancy in experience between the two surgeons involved in this study (i.e. 10 versus 25 years). However, as stated in their letter, surgical intervention for FAI has gained mainstream acceptance only as recently as the turn of the century. Regardless of the existence or otherwise of a learning curve, both of these surgeons have therefore had similar time periods in order to become familiar with this entity and its various surgical indications. We are therefore of the opinion that this discrepancy in surgical experience has little impact upon our reported findings.

In response to Drs. Beaulé's and Rakhra's specific questions relating to the activity of each surgeon, the breakdown is as follows: 78 patients were included in the study group, 39 designated as having cam-type FAI and 39 as not having this condition. Of the affected group, 14 underwent trochanteric osteotomy and osteochondroplasty,

D. G. Lohan $(\bowtie) \cdot$ L. L. Seeger $\cdot$ K. Motamedi $\cdot$ S. Hame

J. Sayre

David Geffen School Of Medicine At UCLA,

Los Angeles, CA, USA

e-mail: derek.lohan@gmail.com
14 underwent osteochondroplasty alone and 1 underwent arthroscopic debridement. All 39 patients in the FAInegative group underwent arthroscopy, no open procedures were performed in this group. Of course, it should be borne in mind that the decision to proceed with open or arthroscopic evaluation was based upon a combination of patient history, symptomatology and imaging findings, in addition to perhaps other unknown contributing factors. This is a limitation inherent to the retrospective design of any study, a limitation that was discussed at length in our paper. It is likely that this distribution of procedures reflects selection of those considered most likely to warrant surgical correction for an open procedure, while those with atypical symptoms or imaging findings were evaluated with arthroscopy. As a result of only one patient having undergone arthroscopic femoral osteochondral debridement, correlation between type of surgical procedure and alpha angle measurement cannot be assessed.

It seems that Drs. Beaulé and Rakhra have misinterpreted the use of clinical impingement throughout our study. In not a single instance do we use or advocate the use of a positive clinical impingement test as the gold standard for the presence or absence of FAI. Rather we simply evaluated the potential use of this rapidly and routinelyperformed clinical examination in suggesting the presence or absence of this condition and compared its performance with that of the alpha angle, AFD (anterior femoral distance) and FNR (femoral neck ratio). It is widely recognized that a positive clinical impingement test is quite non-specific, merely suggesting the presence of acetabular rim "disease." It is our opinion, however, that it speaks volumes that this clinical test out-performed alpha-angle measurement in suggesting the presence or absence of camtype FAI in the current study. 
Eijer et al. in 2001 described a method for the measurement of anterior head-neck offset and anterior head-neck offset ratio on cross-table lateral radiographs and evaluated the potential of these measurements in the screening of symptomatic patients for anterior FAI [2]. In contrast to the technique proposed by those authors (which employed the anterior aspect of the femoral head as one reference point), we concentrated upon the depth of epiphyseal overgrowth at the femoral head-neck junction in the derivation of our measurements. We agree entirely with Drs. Beaulé and Rakhra that normalized ratios are worthy of evaluation in this instance. It was this reasoning that led to our use of the FNR in this study, a normalized measure of the AFD with respect to the femoral neck diameter at the level of epiphyseal overgrowth.

We welcome the opportunity to address Drs. Beaulé's and Rakhra's concern regarding our interpretation of the alpha angle measurement. These readers state that in the original description Notzli et al. [3] "point to the subchondral bone as the reference point for the alpha angle measurement." We note, however, that in contradiction to this statement, Notzli et al. defined the "anterior extent of the concavity of the femoral neck" as a "point where the distance from the bone to the centre of the head first exceeds the radius of the cartilage-covered head." Thus, Notzli et al. specify inclusion of the femoral articular cartilage in drawing a circle encompassing the femoral head, as in our illustrated cases.

Finally, we must disagree with Drs. Beaulé's and Rakhra's interpretation of our explanation for why our results differed so significantly from those of Notzli et al. and Kassarjian et al. [4]. Again, we did not "state that this was biased because of the more advanced head-neck overgrowth present in those two papers." Rather we suggested that this difference in findings may be explained "at least in part" by selection bias, given that patients were selected for inclusion in those studies based upon the presence of positive clinical impingement tests. We at no time assume a dynamic process as queried by Drs. Beaulé and Rakhra. Rather we suggest that the inclusion techniques employed in these two papers may have resulted in preferential selection of patients with greater degrees of femoral head-neck offset (i.e. obviously abnormal clinical impingement tests).

To finish we must disagree with the suggestion of Drs. Beaule and Rakhra that we alter the conclusions of our paper. While studies exist in the literature supporting the reliability of this technique, so too do studies raising concern regarding its clinical utility [5]. We add our paper to the latter group. Our paper did not show that "the alpha angle measurement value will vary depending which MRI imaging plane is used" as suggested by the letter-writers. Rather we support the true findings of this statistical study, namely that the alpha angle performed poorly in our evaluated patient population and was of no value in suggesting the presence or absence of cam-FAI. Of the evaluated parameters (alpha angle, AFD, FNR, and clinical impingement test), the clinical impingement test was shown to be the most reliable predictor of the presence of cam-FAI.

Open Access This article is distributed under the terms of the Creative Commons Attribution Noncommercial License which permits any noncommercial use, distribution, and reproduction in any medium, provided the original author(s) and source are credited.

\section{References}

1. Lohan DG, Seeger LL, Motamedi K, Hame S, Sayre J. Cam-type FAI: is the alpha angle the best MR arthrography has to offer? Skeletal Radiol. 2009;38:855-62.

2. Eijer H, Leunig M, Mahomed MN, Ganz R. Cross-table lateral radiographs for screening of anterior femoral head-neck offset in patients with femoro-acetabular impingement. Hip Int. 2001;11:38-41.

3. Notzli HP, Wyss TF, Stocklin CH, Schmid MR, Treiber K, Hodler J. The contour of the femoral head-neck junction as a predictor for the risk of anterior impingement. J Bone Joint Surg Br. 2002;84:556-60.

4. Kassarjian A, Yoon LS, Belzile E, Connolly SA, Millis MB, Palmer WE. Triad of MR arthrographic findings in patients with cam-type femoroacetabular impingement. Radiology. 2005;236 (2):588-92.

5. Nouh MR, Schweitzer ME, Rybak L, Cohen J. Femoroacetabular impingement: can the alpha angle be estimated? Am J Roentgenol. 2008;190:1260-2. 